\title{
German Medical Informatics Initiative
}

\section{A National Approach to Integrating Health Data from Patient Care and Medical Research}

\author{
Sebastian C. Semler'; Frank Wissing'ㄹ Ralf Heyder ${ }^{3}$ \\ ${ }^{1} T M F$ - Technology, Methods and Infrastructure for Networked Medical Research, Berlin, Germany: \\ ${ }^{2} \mathrm{MFT}$ - German Association of Medical Faculties, Berlin, Germany; \\ ${ }^{3}$ VUD - German Association of Academic Medical Centers, Berlin, Germany
}

\begin{abstract}
Keywords
Medical informatics, data integration, secondary use of clinical data, data integration, research data infrastructure, interoperability, patient consent, data sharing, national coordination
\end{abstract}

\section{Summary}

This article is part of the Focus Theme of Methods of Information in Medicine on the German Medical Informatics Initiative. The Medical Informatics Initiative (MII) was launched within the scope of the German Federal Ministry of Education and Research's (BMBF) Medical Informatics Funding Scheme, with the goal of developing infrastructure for the integration of clinical data from patient care and medical research in Germany. Its work is to be performed over the course of a decade (2016-2025) across three funding phases, with the first two concentrating on university hospitals. During the

Correspondence to:

Sebastian Claudius Semler

TMF - Technologie- und Methodenplattform für die

vernetzte medizinische Forschung e.V.

Charlottenstrasse 42

10117 Berlin, Germany

Tel.: +49 - (0)30 - $2200247-0$

Fax: $+49-(0) 30-2200247-99$

E-mail: sebastian.semler@tmf-ev.de conceptual phase (now concluded), a central supporting project ensured coordination and laid the ground for standardised solutions for all the initiative's sites and scientific consortia that will enable effective data use and exchange, both for health care as well as research. The conceptual phase focused on the following: a) interoperability, through the consistent use of international standards (from an early stage, i.e. primary IT systems in patient care); b) standardised templates for patient consent and harmonised data protection; and c) standard rules for data use and access (monitoring and safeguarding access to data). On this basis, the initiative aims in the long term to improve medical research (particularly health care research, using data from treatments), to accelerate the transfer of knowledge from research to patient care - and to provide important impetus for the digitalization of medicine in Germany.

Methods Inf Med 2018; 57(Open 1): e50-e56 https://doi.org/10.3414/ME18-03-0003 received: May 18, 2018 accepted: May 18, 2018

\section{Introduction}

On 16 November 2015, the German Federal Ministry of Education and Research (BMBF) published its Medical Informatics Funding Scheme [1] and a corresponding call for proposals - underlining that the
German federal government recognises digitalization's growing role in health care, and the potential to improve diagnostics and treatment through greater adoption of IT in medical research. The Medical Informatics Funding Scheme has multiple goals:
- to harness the opportunities created by digitalization in medicine,

- to develop innovative IT solutions to improve patient care and research,

- to support and advance the exchange and use of data across the boundaries of individual institutions and sites,

- in particular, to increase the exchange and use of data between health care and clinical/biomedical research,

- to accelerate and improve the availability of research findings for health care through greater electronic exchange of knowledge and data,

- and to expand the skillset needed to make these goals a reality, and to strengthen education, training and professional development in medical informatics.

To achieve these aims, the Medical Informatics Initiative (MII) called for the formation of scientific consortia with the aim of designing and implementing innovative IT solutions based on specific use cases, and to ensure the interoperability of these solutions across consortia.

The funding scheme comprises three phases ( $\$$ Figure 1 ):

- Conceptual phase 2016-2017

- Development and networking phase 2018-2021

- Consolidation and further development phase 2022-2025

The MII funding scheme will initially focus on German university hospitals and medical centres. These facilities treat a total of approximately 1.8 million patients annually, representing 10 per cent of all inpa- 


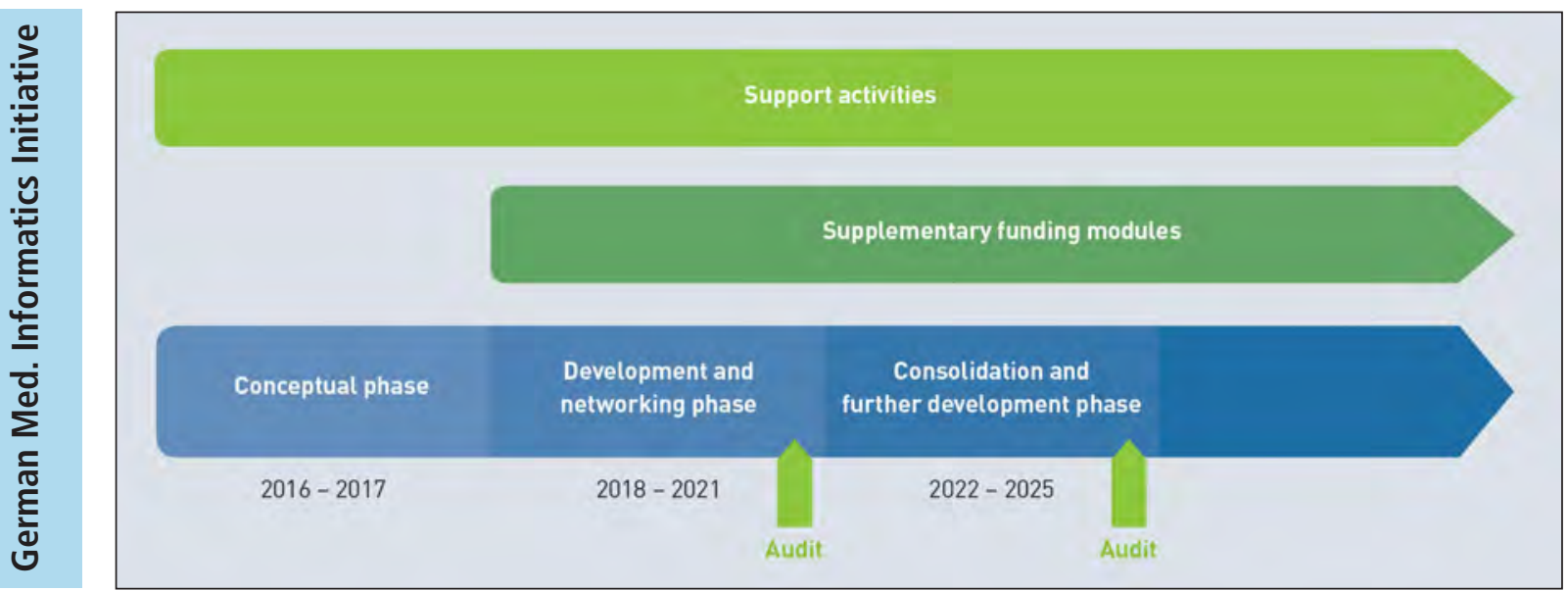

Figure 1 The phases of the Medical Informatics Initiative: funding is provided to the scientific consortia over three successive phases (the nine-month conceptual phase, four-year development and networking phase, and four-year consolidation and further development phase). There are also concurrent activities (support activities, supplementary funding modules). (Modified from [1]).

tient cases in Germany. They are therefore highly relevant to patient care, tightly integrated with medical research on an internationally competitive level, and a significant starting point for the Medical Informatics Initiative. However, the initiative is expected to generate impetus for and benefits to many aspects of the health care system and the health care industry.

In addition to scientific consortia, the funding scheme foresees a supporting project that provides assistance and coordination. This is led by the National Steering Committee (NSC), comprising representatives from the funded consortia. Furthermore, the NSC is supported by the coordination office organised and operated by TMF (Technology, Methods and Infrastructure for Networked Medical Research), MFT (German Association of Medical Faculties), and VUD (German Association of Academic Medical Centers).

A key aim of the initiative is the collection, integration and use of data resources to improve patient care. This is highly relevant for Germany, where data resources and responsibilities have traditionally been fragmented and siloed (health care vs research, outpatient vs inpatient care, regulatory decision making at federal vs state level, etc.), and have proved to be a barrier to achieving a competitive degree of digitalization. The German Medical Informatics Initiative and its funding scheme provide a good opportunity to strengthen medical informatics at German universities, and to promote the deployment of advanced IT solutions in health care at the national level [2]. In particular, it aims to provide fresh impetus for standardisation and interoperability across the boundaries of various fields of health care, individual German states, and specific projects making an important contribution to the development of e-health in Germany.

\section{The Conceptual Phase 2016-2017: A Competition of Ideas, a Governance Structure for Collaboration and Interoperability}

The conceptual phase had two objectives. First, consortia were to be established that, following an initial external assessment, developed competing ideas and solutions for the establishment of data integration centres (DICs) and innovative use cases. At the end of the nine-month phase (1 August 2016 to 30 April 2017), these were subject to international evaluation in order to select the most attractive solutions for implementation. Second, the conceptual phase was designed to ensure collaboration across all participating sites and consortia from an early stage, laying the foundations for their coordination and interoperability across Germany. The National Steering
Committee (NSC) defined key parameters (key issues) to be referenced by all consortia applications and solutions. To this end, an agenda was agreed and the action items defined within the scope of an 18-month supporting project (1 July 2017 to 31 December 2018). This project identified issues where agreement is needed at the national, cross-consortia level from early on.

The corresponding decision-making process was defined in the National Steering Committee's bylaws (Rules of Procedure) [3]. D Figure 2 illustrates the governance structures and their relationships: the National Steering Committee (NSC) comprises representatives of the consortia and the central coordination office. The consortia's NSC nominees include medical informatics experts, and representatives of the management boards of university hospitals and the deans of medical faculties, to ensure outcomes and decisions are implemented at participating sites. The NSC is supported and accompanied by a) the central coordination office, b) the German Federal Ministry of Education and Research $(\mathrm{BMBF})$ and by its project management agencies, c) the dialogue forum, which gives stakeholders an opportunity to influence the workings of the initiative over and above its purely research goals. The NSC holds an annual general meeting, while its working groups (which can, in turn, establish their own substructures) meet on a regular basis to prepare docu- 
Figure 2

The governance structure of the German Medical Informatics Initiative: the National Steering Committee (NSC) and its working groups (and task forces - selected examples are shown here), the central coordination office, the annual general meeting, and the dialogue forum for engagement with stakeholders. The German Federal Ministry of Education and Research (BMBF) provides funding, plus support in the shape of its project management agencies, for the NSC and the coordination office.

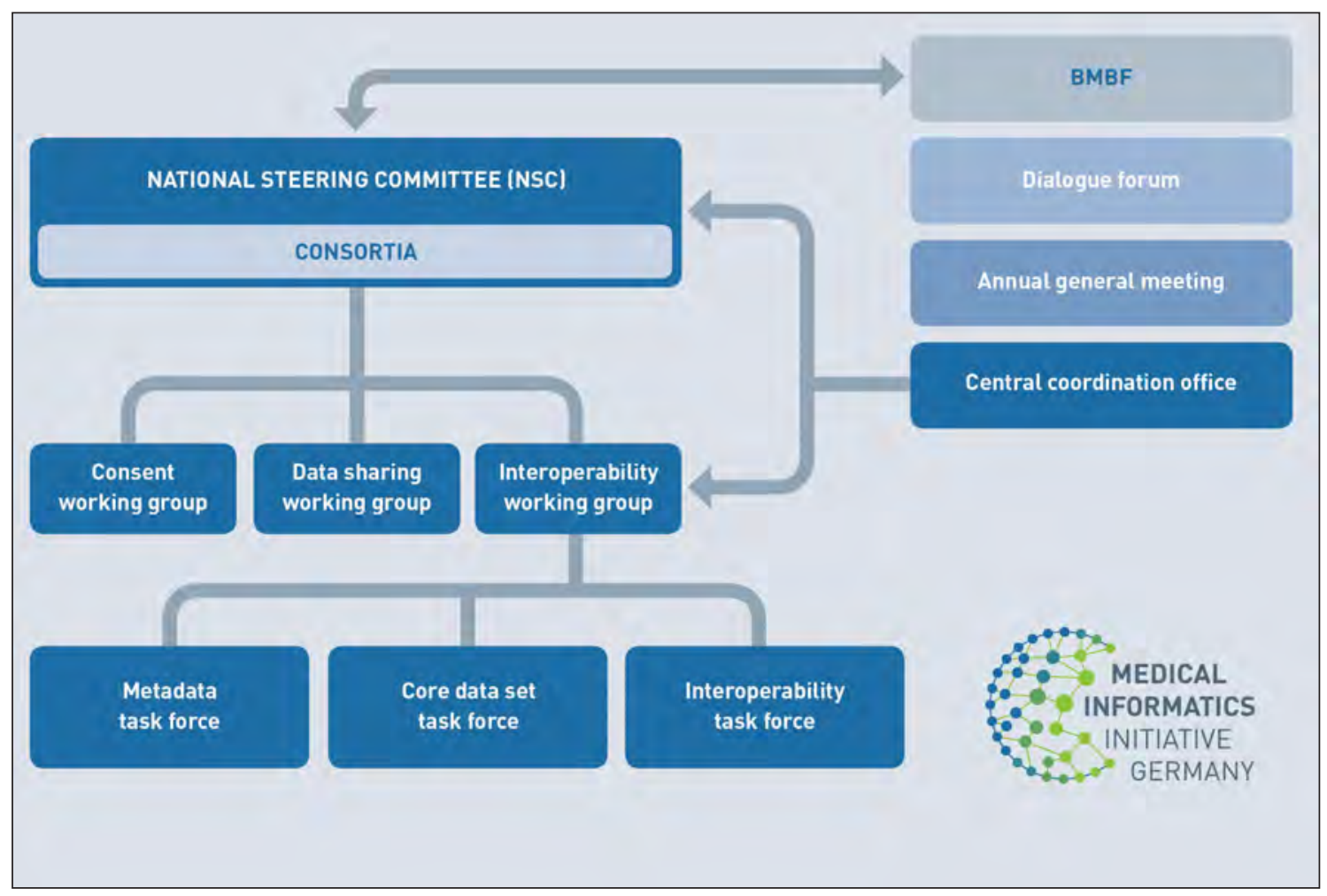

ments for decision-making - e.g. to safeguard collaboration across consortia and to ensure the data integration centres are capable of cooperating at an international level. All university hospital sites in Germany can take part in the working groups; external experts and institutions are invited to participate where appropriate (including, for example, standardisation bodies such as HL7, IHE). Moreover, existing entities and structures, particularly from the MII's three supporting associations (TMF, MFT, VUD), are included to avoid duplication of effort (and include e.g. the TMF's data protection, IT and quality management and scientific communications working groups).

Nearly all German university hospitals participated in the initiative's conceptual phase, in a total of seven consortia (see - Figure 3): AgenDa Medical Informatics for Healthcare, Research \& Education (ADMIRE), [The Munich-Tübingen Alliance for] Data Integration and Future Medicine (DIFUTURE), Health Data for Care and Research (HD4CR), HeidelbergGöttingen-Hannover Medical Informatics (HiGHmed), Medical Informatics for Research and Care in University Medicine
(MIRACUM), Synergistic Health Data Access for Research and CarE - Innovation and Translation (share-it!), and Smart Medical Informatics Technology for Health Care (SMITH). Almost all consortia have participants drawn from across Germany, not just individual states, and work towards their defined goals in a spirit of cooperation in line with their professional expertise, with no regional focus. The positive response to the BMBF's call for proposals and the nationwide, cooperative character of the consortia led to one of the initiative's early successes: when additional partners are taken into account, the MII secured the participation of nearly all German university hospitals, and a total of 15 of the 16 German federal states. This highlights the nationwide relevance of the initiative, as well as the opportunity it provides to increase standardisation across sites and states. Furthermore, this underscores the challenge associated with cooperating and coordinating activities across multiple partners and sites, and also the need to consider and comply with all relevant legal requirements at state level (e.g. legislation governing data protection or hospital management) from the initiative's outset.

\section{Roadmap, Work and Results of the Medical Informatics Initiative Working Groups During the Conceptual Phase}

The work and decisions of the National Steering Committee (NSC) and its various working groups during the conceptual phase focused on three key topics:

- Interoperability

- Patient consent and data protection

- Data use and access rules

The NSC only defined those requirements and parameters that were essential to the express goal of the funding scheme, i.e. to ensure data exchange and shared use nationwide, and across sites and consortia, in accordance with standardised access criteria and data security mechanisms. The consortia retain a large degree of freedom when it comes to the use cases and infrastructure while competing during the conceptual phase.

On this basis, the NSC agreed on a roadmap for the Medical Informatics Initi- 
I - University of Cologne

DIFUTURE

Elechnical University of Munich

(TUM)

- University of Augsburg

- Ludwig Maximilian University (LMU)

- University of Tübingen

HDLCR

- Charité University Hospital Berlin

- Ulm University Medical Center

- University Hospital of Würzburg

HiGHmed

a Heidelberg University Hospital

- University Medical Center Göttingen

- Hanover Medical School

- German Cancer Research Centre,

Heidelberg

MIRACUM

- Friedrich Alexander University

Erlangen-Nuremberg

- Goethe University Frankfurt

- Medical Faculty of the University of Freiburg

- Justus Liebig University Giessen

- German Cancer Research Centre,

Heidelberg

- Heidelberg University

- University Medical Center of the

Johannes Gutenberg University Mainz

- Philipps University Marburg

share-it!

- Greifswald University Hospital

- Technical University of Dresden and

University Hospital Carl Gustav Carus

- University Medical Center of

Hamburg-Eppendorf

- University Medical Center

Schleswig-Holstein

- offIS e.V, Oldenburg

SMITH

- Leipzig University

- RWTH Aachen University

- University Hospital Jena

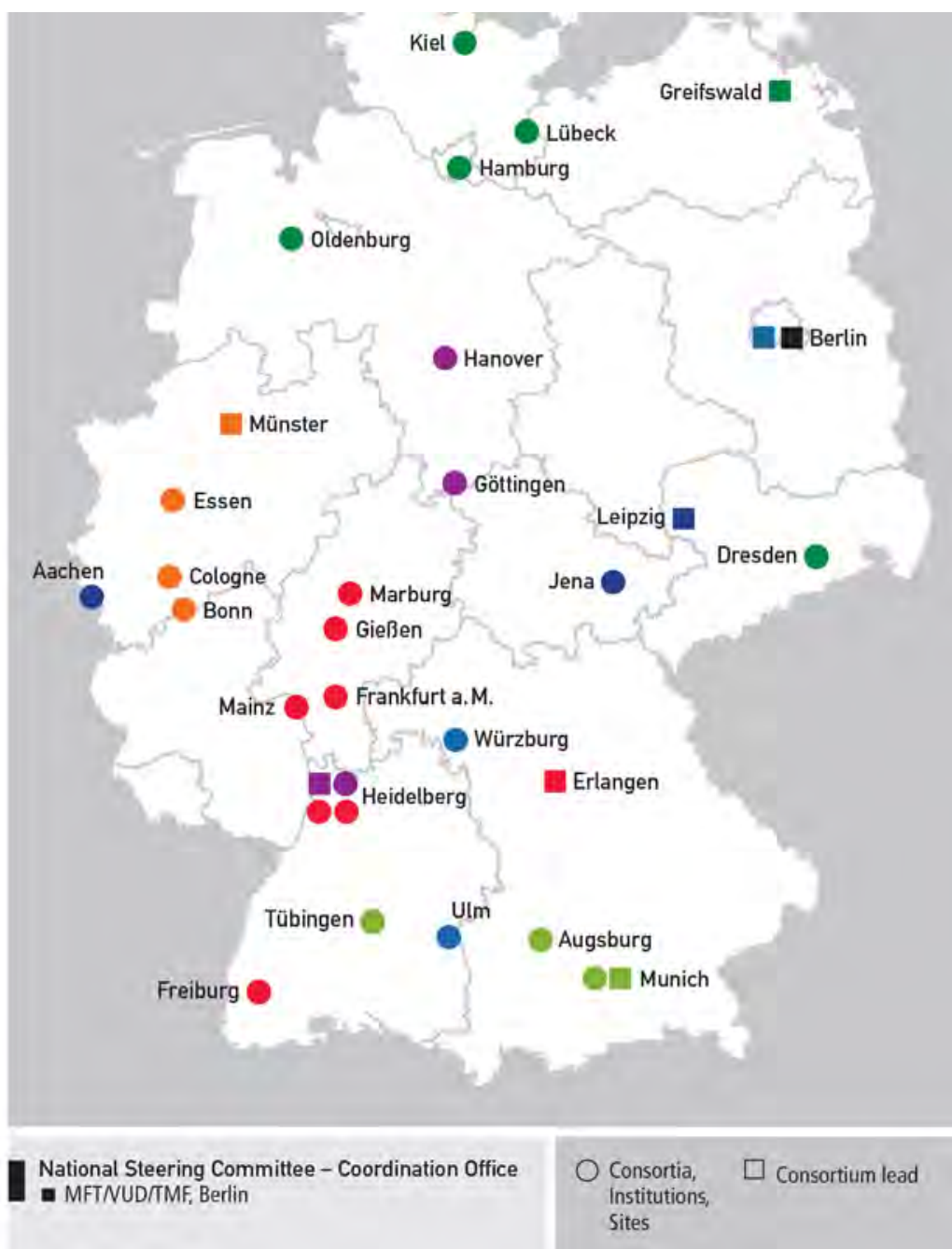

Figure 3

The seven consortia and their participating sites during the conceptual phase

2016-17 are shown on the map (only the consortia's university sites - not including non-university and industry partners). ative. It defines a number of steps for the harmonisation and development of shared infrastructures, including: (1) standards for and technical implementation of electronic patient consent declarations, (2) definition of tasks and the role of trusted third parties for identity management, (3) rules and technical support for data use and access committees, (4) data protection solutions, agreed with the corresponding oversight agencies, (5) semantic interoperability (core data set, value sets, use of international terminology) and meta-data services, (6) methods and portals for data sharing (central registration point for data access requests and projects), (7) audit criteria and shared use cases, (8) new concepts for patient involvement and empowerment, (9) activities to strengthen research, education and professional development in medical informatics, (10) and roll-out and further integration (see $[4,5$, $6,7])$. International experiences and examples (e.g. PCORnet [8], OHDSI [9]) were incorporated into the development of strategies and the roadmap.

An essential step on this roadmap was a paper summarising key parameters for interoperability, completed during the conceptual phase [10]. The paper describes a voluntary commitment to fulfil minimum interoperability requirements. This includes the standardisation of data and processes, and the establishment of interoperable IT infrastructures. Furthermore, this takes into account the technical processes and components needed to implement patient consent and data protection. The minimum interoperability requirements have been described and will be updated as the project progresses.
Key to interoperability is the definition of a core MII data set. This describes the minimum data to be transferred from the primary patient care system to the data integration centres (DICs) for each individual patient case - all in standardised, pseudonymised form for use in future research [11]. The core data set will be expanded step-by-step in modules, in accordance with specific criteria: (a) relevance to research and patient care, (b) relevance to the consortia's use cases, (c) availability and accessibility at the MII sites, (d) the degree to which the data is structured, and (e) the availability of terminology. The modules are described (see $>$ Figure 4) [12], and their detailed specifications and implementation will be agreed in the MII interoperability working group and published in future. Data for the first modules are already available in standardised form within 
Germany (e.g. ICD-10- or OPS-encoded diagnoses and procedures); data for the next modules are already being prepared (e.g. comprehensive standardisation of a subset of lab data with LOINC, preparation of medication data using standardised medication catalogues). This work has a direct impact on the standardisation and improved quality of data in primary IT systems, with benefits for patient care.

The initiative's greatest challenge after interoperability is data protection. In Germany, the corresponding oversight agencies, applicable legislation and their interpretation vary from state to state (as illustrated in $>$ Figure 5 [13]). Consequently, it is important to define standard procedures and approval mechanisms across sites, even within a single consortium. Cross-site, long-term research on patient care data can only be conducted lawfully with informed patient consent. With this in mind, the MII consent working group developed a template form for patient information and consent. The aim is to standardise key elements of the consent form, therefore enabling data to be used by multiple parties. It comprises a number of predefined modules to which the patient can agree, allowing their data to be made available for future medical research. In 2017, the template was reviewed, edited and agreed with the Association of Medical Ethics-Committees in Germany (AK-EK). Discussions with data protection oversight agencies for the German states and the federal government are currently ongoing (2018). Furthermore, efforts are being made to define a generic data protection concept that would allow patient care data to be used in research. The content of this concept and the process of finding agreement with Germany's data protection oversight agencies build on Germany's established templates, resources and processes, which were jointly developed with TMF (see [14]).

A third, crucial topic that needed to be considered early on in decision-making was how to establish mechanisms for standardising data access across sites and consortia in accordance with defined data protection, scientific and ethical criteria. The initiative's data sharing working group developed a detailed paper that clearly defines the key elements of future use and ac-

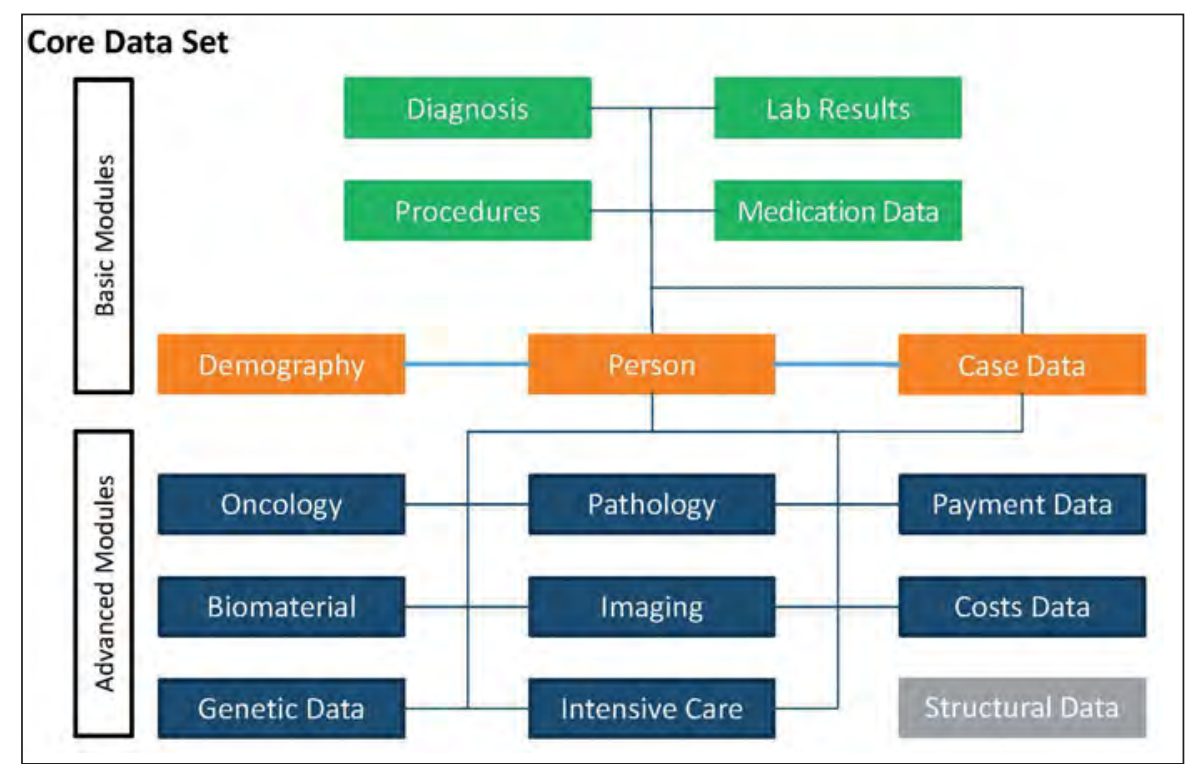

Figure 4 This diagram outlines the modules of the MII core data set. These will be made available in stages as data from research and health care are integrated. The basic modules include administrative (orange) and medical (green) data elements that can be made available in harmonised form relatively quickly. More complex data elements, which require more extensive preparation and harmonisation, are shown in the advanced modules (blue). In addition to data on specific patient cases, it is also possible to aggregate structural data of a general nature (e.g. on the institution providing treatment) (structural data, grey).

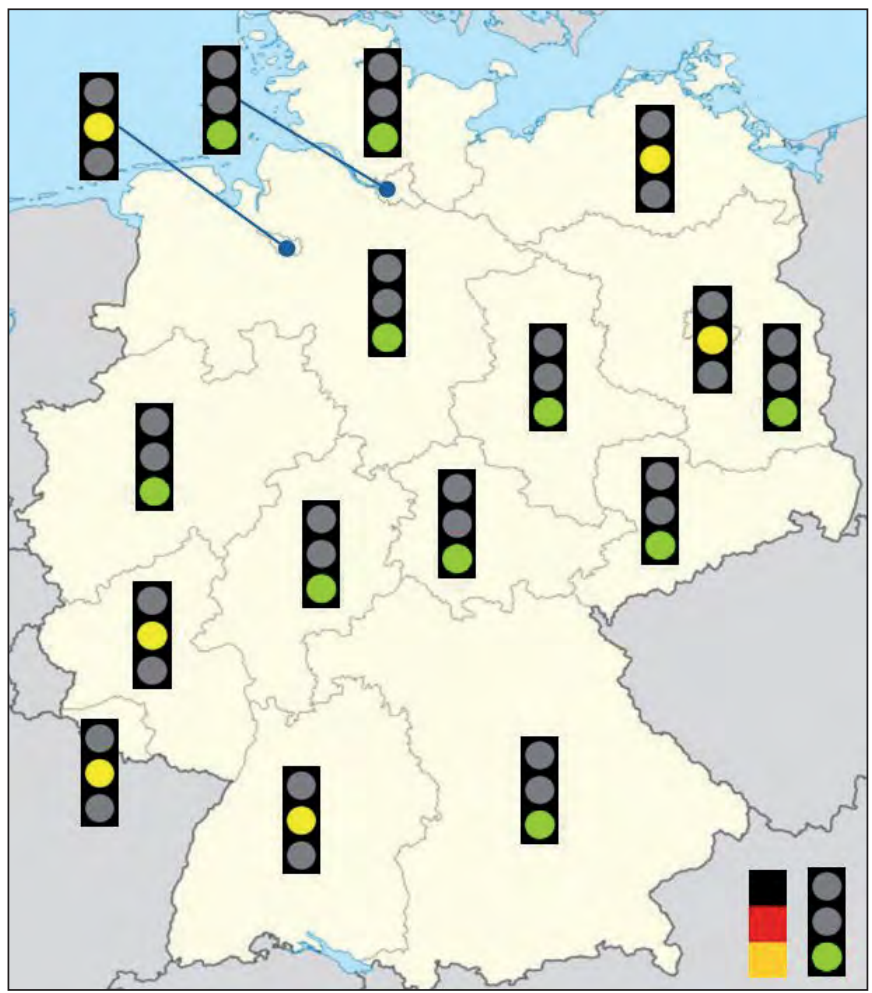

Figure 5 This illustrates the various legislative environments in each of Germany's 16 states and at federal level - with regard to whether and to what extent research can use treatment data, and whether informed patient consent is required. The traffic lights indicate each state's legal situation: a) permissible with no restrictions (green) with consent; consent limited to an individual case or specific research project is not required; b) permissible with restrictions (yellow), where consent must be limited to an individual case or specific research project. (Similar differences between the German states have also been observed for other relevant parameters) (see [13]). 


\section{Consortia and participants} during the development and networking phase

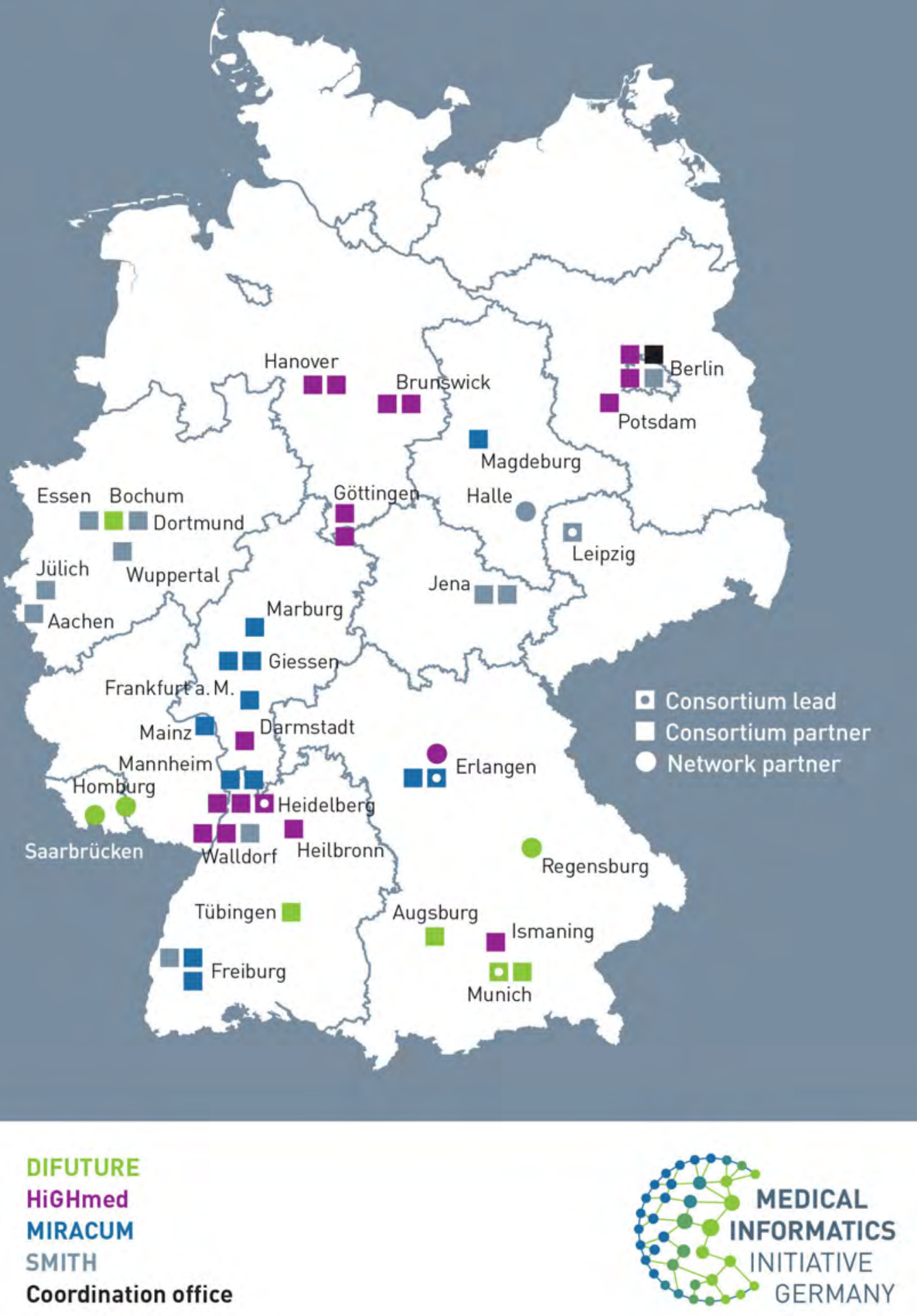

Figure 6

The sites of the four consortia chosen for funding during the development and networking phase 2018-2021 are shown on the map (including additional university, non-university and industry partners). Universities from discontinued consortia that were active during the conceptual phase can join these four consortia by 2019 (see -Figure 3; not depicted here). 
cess, based on extensive experience from large cohort studies. Its specifications include: (a) use and access committees at all data integration centres, with transparent ruling on data use requests, (b) transfer offices at all data integration centres that guarantee secure data transfer from a technical perspective, and (c) a central, independent coordination and registration office that receives and manages central data requests, and that ensures transparency regarding the purpose and achievements of data use vis á vis the general public and stakeholders [15].

\section{Outlook: The Start of the Development and Net- working Phase 2018-2021}

The above specifications and requirements developed jointly by all sites formed the basis for solutions and funding applications. These were evaluated in July 2017, and four consortia were selected for further funding totalling 120 million euros: DIFUTURE, HiGHmed, MIRACUM, and SMITH (see Figure 6). An additional 30 million euros will be provided for the integration of the other sites into these remaining consortia. This will help ensure the almost nationwide involvement of university hospitals in the Medical Informatics Initiative by 2019 .

Since January 2018 (during the development and networking phase), progress has been made with cross-consortia integration. Ideas and solutions are being evolved in line with the roadmap, and new specifications and requirements defined to ensure interoperability and data usage compliant with applicable legislation. Members of the Medical Informatics Initiative have also begun the first stages of dialogue with the German Federal Ministry of Health and with gematik $\mathrm{GmbH}$, with the aim of enhancing coordination and integration with the telematics infrastructure for patient care in Germany. Going forward, the MII is expected to provide fresh impetus for research infrastructures, standardisation in health care, and the implementation of interoperable electronic patient records - and to close the gap between medical research and patient care, to the benefit of patients and citizens.

\section{References}

1. Federal Ministry of Education and Research [Bundesministerium für Bildung und Forschung, BMBF]. Medical Informatics Funding Scheme: Networking Data - improving health care. BMBF, Oct. 2015 [cited 2018 Jan 31]. Available from: https://www.bmbf.de/pub/Medical_Informatics_ Funding_Scheme.pdf.

2. Semler SC, Wissing F, Heyder R: Förderprogramm Medizininformatik: Quantensprung für Qualität und E-Health. In: Schug SH, Schmücker P, Semler SC, Seidel C, editors. E-Health-Rahmenbedingungen im europäischen Vergleich: Strategien, Gesetzgebung, Umsetzung. (Tagungsband zur TELEMED 2016 - 21. Nationalen Forum für Gesundheitstelematik und Telemedizin). AKA 2016: 31-33. In German.

3. Rules of Procedure for the National Steering Committee of the German Medical Informatics Initiative [cited 2018 Apr 25]. Available from: http://www.medizininformatik-initiative.de/en/ about-initiative/results.

4. Roadmap for the Medical Informatics Initiative 2017-2025 [cited 2018 Apr 25]. Available from: http://www.medizininformatik-initiative.de/en/ about-initiative/results.

5. MII Roadmap for Strengthening Research, Teaching, and Continuing Education [cited 2018 Apr 25]. Available from: http://www.medizininforma tik-initiative.de/en/about-initiative/results.

6. Data Sharing Working Group Audit Approach in Connection with the Medical Informatics Initiative [cited 2018 Apr 25]. Available from: http://www.medizininformatik-initiative.de/en/ about-initiative/results.

7. MII Metadata on Data Availability, Analysis Opportunities, and Cooperation Options [cited 2018 Apr 25]. Available from: http://www.medizinin formatik-initiative.de/en/about-initiative/results.

8. Fleurence RL, Curtis LH, Califf RM, Platt R, Selby JV, Brown JS. Launching PCORnet, a national patient-centered clinical research network. J Am Med Inform Assoc 2014; 21: 578-582.

9. Hripcsak G, Duke JD, Shah NH, Reich CG, Huser V, Schuemie MJ, et al. Observational Health Data Sciences and Informatics (OHDSI): Opportunities for Observational Researchers. Stud Health Technol Inform 2015; 216: 574-578.

10. Joint Interoperability Key Issues Paper [cited 2018 Apr 25]. Available from: http://www.medizinin formatik-initiative.de/en/about-initiative/results.

11. MII Core Data Set [cited 2018 Apr 25]. Available from: http://www.medizininformatik-initiative.de/ en/about-initiative/results.

12. Ganslandt T, Boeker M, Löbe M, Prasser F, Schepers J, Semler SC, Thun S, Sax U. Der Kerndatensatz der Medizininformatik-Initiative: Ein Schritt zur Sekundärnutzung von Versorgungsdaten auf nationaler Ebene. mdi 1/2018: 17-21. In German.

13. Schneider UK. Sekundärnutzung klinischer Daten - Rechtliche Rahmenbedingungen. TMF-Book Series, Vol.12. Berlin: MWV; 2015.

14. Pommerening K, Drepper J, Helbing K, Ganslandt T. Guideline for Data Protection in Medical Research Projects - TMF's generic solutions 2.0. TMF-Book Series, Vol.11. Berlin: MWV; 2014.

15. Data Sharing Working Group - Uniform Use and Access Policy Key Issues Paper [cited 2018 Apr 25]. Available from: http://www.medizininforma tik-initiative.de/en/about-initiative/results. 